\title{
Detection and clinical significance of circulating tumor cells in patients with nasopharyngeal carcinoma
}

\author{
ZUNBEI WEN $^{1}$, ZHONGTAI LI $^{1}$, PANGXIAO YONG ${ }^{1}$, DUNBO LIANG ${ }^{1}$, DI XIE ${ }^{1}$, \\ HAIWEN CHEN $^{1}$, YONGYING YANG ${ }^{1}$, SHIYANG WU ${ }^{2}, \mathrm{CONG} \mathrm{LI}^{2}$ and ZHEN CHENG ${ }^{2}$ \\ ${ }^{1}$ Department of Radiotherapy, The People's Hospital of Gaozhou, Gaozhou, Guangdong 525200; \\ ${ }^{2}$ SurExam Bio-Tech, Guangzhou Technology Innovation Base, Guangzhou, Guangdong 510000, P.R. China
}

Received May 17, 2017; Accepted September 28, 2018

DOI: $10.3892 / 01.2019 .10560$

\begin{abstract}
Nasopharyngeal carcinoma (NPC) is the most common cancer type originating in the nasopharynx, and varies notably from other cancer types of the head and neck in its occurrence, causes, clinical behavior and treatment. Significant effort has been made into understanding the biological properties of circulating tumor cells (CTCs), with previous studies demonstrating the critical role CTCs serve in the metastatic spread of carcinoma. However, associations between NPC and CTCs have not been completely elucidated. Therefore, in the present study, the CanPatrol ${ }^{\mathrm{TM}}$ CTC-enrichment technique and classical in situ hybridization assay were utilized to acquire, identify and classify CTCs from patients with NPC. Subsequently, the correlation between CTCs and the clinical indexes, progression-free survival (PFS), N-cadherin gene expression and the response to therapy were investigated. The present study then determined whether the Wnt/ $\beta$-catenin signaling pathway served a role in therapy for NPC cells. Collectively, the research demonstrated that CTCs could be detected in patients with NPC. Additionally, CTCs exhibited a statistically significant association with the Epstein-Barr virus infection prior to therapy and Eastern Cooperative Oncology Group score following therapy. Furthermore, co-treatment with cisplatin and paclitaxel significantly decreased the number of CTCs. In addition, mesenchymal CTCs may serve
\end{abstract}

Correspondence to: Professor Zunbei Wen, Department of Radiotherapy, The People's Hospital of Gaozhou, 89 XiGuan Road, Gaozhou, Guangdong 525200, P.R. China

E-mail: zunbeiwen118@163.com

Abbreviations: NPC, nasopharyngeal carcinoma; CTCs, circulating tumor cells; ISH, in situ hybridization; PFS, progression-free survival; EBV, Epstein-Barr virus; ECOG, Eastern Cooperative Oncology Group; EMT, epithelial-mesenchymal transition; CR, complete response; $\mathrm{PR}$, partial response; $\mathrm{SD}$, stable disease; $\mathrm{PD}$ progressive disease

Key words: nasopharyngeal carcinoma, circulating tumor cells, $\mathrm{N}$-cadherin, CanPatrol ${ }^{\mathrm{TM}}$ CTC-enrichment technique, Wnt $/ \beta$-catenin signaling pathway as a predictor of PFS. Finally, the present study demonstrated that cisplatin combined with paclitaxel induced apoptosis and decreased the tumor markers in NPC cells through the Wnt/ $\beta$-catenin signaling pathway. In conclusion, these data indicated that CTCs may serve as a biomarker in monitoring the therapeutic efficacy of treatments for NPC. Furthermore, the Wnt/ $\beta$-catenin signaling pathway served a therapeutic role in the treatment of NPC.

\section{Introduction}

Human nasopharyngeal carcinoma (NPC) is an invasive and metastatic head and neck cancer, which was reported to be prevalent in Southeast Asia, particularly in the south of China (1). It originates from the nasopharynx epithelium and has a complicated pathogenesis (2). Certain geographical locations, including southern China, and ethnic groups, including Asian, have been reported to affect the predisposition of the populace to NPC (3). It has been reported that factors, including the Epstein-Barr virus (EBV) infection, an unhealthy diet and smoking, may contribute to the incidence of NPC $(4,5)$. Although treatments of radio and adjuvant chemotherapy for NPC can prolong the lifespan of patients, further research and therapeutic methods pertaining to NPC are required.

Circulating tumor cells (CTCs), which were first identified in 1869 by Ashworth (6), flow into the peripheral blood system from original and metastatic tumors, and lay the foundation for tumor metastasis (7). To undertake tumor development, CTCs undergo a process termed epithelial-mesenchymal transition (EMT), which serves a key role in tumor metastasis $(8,9)$. CTCs are generally classified into three varieties: Epithelial CTCs, mesenchymal CTCs and hybrid CTCs (10). Of these subtypes, the most migratory and invasive of these CTCs are the mesenchymal CTCs (11). Therapeutically, CTCs are frequently defined as a 'liquid biopsy' specimen, which is a snapshot of tumor cells in the circulation at a specific point in time (12). The presence and detection of CTCs are able to offer significant information regarding the diagnosis and treatment of certain types of cancer with low survival rates (13-19). Nevertheless, there is a lack of sufficient research regarding CTCs of NPC.

$\mathrm{N}$-cadherin is a hemophilic transmembrane cell adhesion molecule (20). The inappropriate expression of $\mathrm{N}$-cadherin 
is an indicator of EMT, which is associated with tumor malignancy and metastasis (21). According to a study by Nieman et al (22), N-cadherin contributes to enhanced tumor cell survival, migration and invasion. Although there is an increasing amount of evidence that supports the association between $\mathrm{N}$-cadherin level and cancer progression (23-25), to the best of our knowledge, the role of $\mathrm{N}$-cadherin on tumor metastasis has not been comprehensively examined.

The Wnt/ $\beta$-catenin signaling pathway serves an important role in the self-renewal and differentiation of cancer cells (26-28); however whether Wnt/ $\beta$-catenin signaling in NPC cells has a role in the effect observed when cisplatin is combined with paclitaxel therapy requires further investigation.

The progressive CanPatrol ${ }^{\mathrm{TM}}$ CTC-enrichment technique and classical in situ hybridization (ISH) assay were utilized to isolate and identify CTCs from patients with NPC. Subsequently, the correlation between multifold CTCs and the clinical stages, clinical parameters, $\mathrm{N}$-cadherin gene and Wnt $/ \beta$-catenin signaling of NPC cells was investigated.

\section{Materials and methods}

Materials. $\mathrm{KHCO}_{3}$, ethylene diamine tetraacetic acid (EDTA), formaldehyde, $\mathrm{NH}_{4} \mathrm{Cl}$ and Tris- $\mathrm{HCl}$ were provided by Sinopharm Chemical Reagent Co., Ltd (Shanghai, China). Horse serum, PBS, DAPI, 0.1X SSC and SDS were purchased from Sigma-Aldrich (Merck KGaA, Darmstadt, Germany). Protease was derived from Qiagen GmbH (Hilden, Germany). The 24-well plates were from Thermo Fisher Scientific, Inc. (Waltham, MA, USA). $\beta$-catenin inhibitor XAV939 was obtained from MedChemExpress (Princeton, NJ, USA). Cell Counting kit- 8 was acquired from Beyotime Institute of Biotechnology (Shanghai, China). EBV nucleic acid quantitative detection reagent kit was from Beijing SinoMDgene Technology Co., Ltd., Beijing, China. Total caspase-3 (cat. no. BS9865M), active caspase-3 (cat. no. BS9872M), B-cell lymphoma 2 (Bcl2; cat. no. BS1511), $\beta$-catenin (cat. no. BS3603), c-Myc (cat. no. BS1245) and GAPDH (cat. no. AP0066) primary antibodies, and horseradish peroxidase-conjugated goat anti-rabbit secondary antibodies (cat. no. BS13278) were obtained from Bioworld Technology, Inc. (St. Louis Park, MN, USA).

Patient samples. The People's Hospital of Gaozhou (Gaozhou, China) recruited 60 patients with NPC for the present study between March 2015 and August2016. Individuals were removed if they had a previous history of cancer or had undergone radiotherapy or chemotherapy at diagnosis. The 60 patients were aged $>18$ years. Among the patients, there were 50 males and 10 females. From these, 45 people were $>50$ years old and 15 people were $\leq 50$ years old, with a median age of 42 years (range, 35-67 years). The mean age of all patients at diagnosis was $50.8 \pm 11.3$ years. Additionally, a total of 18 healthy volunteers acted as controls and comprised of 9 males and 9 females. From these, 11 people (5 males and 4 females) were $\geq 50$ years old and 7 people were $\leq 50$ years, with a median age of 41 years (range, 33-64 years). In order to prevent underlying skin cell contamination from the venipuncture for patients with NPC and healthy volunteers, the first $2 \mathrm{ml}$ of blood samples were not used, and $5 \mathrm{ml}$ peripheral blood samples with anticoagulant EDTA $(1.4 \mathrm{mg} / \mathrm{l})$ were gathered. Experiments were performed within $4 \mathrm{~h}$ following the collection of blood samples. Samples were collected three times: Prior to therapy, during therapy and post-therapy. Volunteers only donated samples once.

Ethics approval and informed consent. The present study was approved by the Ethical Committee of The People's Hospital of Gaozhou. The patients with NPC and healthy volunteers provided written informed consent for inclusion in the present study.

Isolation of CTCs by size. The principle of adopting the CanPatrol CTC-enrichment technique to isolate CTCs is based on the attestation from $\mathrm{Wu}$ et al $(29,30)$. Briefly, a filtration method (29) was employed utilizing a standard membrane with 8- $\mu \mathrm{m}$ diameter pores (EMD Millipore, Billerica, MA, USA). To augment the efficiency of filtration, a system was recruited, which was composed of four parts: A filtration tube including the membrane (SurExam Bio-tech, Guangzhou, China), an E-Z 96 vacuum manifold (Omega Bio-Tek, Inc., Norcross, GA, USA), a manifold vacuum plate with valve settings (SurExam Bio-tech) and a vacuum pump (Auto Science, Tianjin, China). In order to prepare for filtration, firstly, the red blood cell lysis buffer (the water solution of $154 \mathrm{mM} \mathrm{NH}_{4} \mathrm{Cl}, 10 \mathrm{mM} \mathrm{KHCO}_{3}$ and $0.1 \mathrm{mM}$ EDTA) was utilized to remove erythrocytes. Secondly, PBS including $4 \%$ formaldehyde was employed to resuspend the remaining cells, for $5 \mathrm{~min}$ at $4^{\circ} \mathrm{C}$. The cell suspension was then placed into the filtration tube, and the pump valve and the manifold vacuum plate valve were turned on in turn. To evaluate if CTCs could be predictors of PFS, the PFS of 60 patients was determined by Kaplan-Meier analysis following a median follow-up time of 8 months (range, 5-21 months).

Tri-color RNA ISH assay. Branched DNA (bDNA) signal amplification technology, which is increasingly used in molecular diagnostics, achieves its goal by signal amplification on the bDNA probe following direct binding of a large hybridization complex to a target sequence $(31,32)$. In brief, it is a compound of multi-step nucleic acid hybridization. Firstly, the target sequences are captured by capture probes, which combine with the bDNA signal amplification probes, and serve a role as a bridge between target sequences and amplification probes. As a result, a branched structure is produced. Finally, fluorescent dye-combined label probes are used to bind to bDNA molecule amplification probes by hybridization (30). Using bDNA signal amplification technology, the present study utilized the RNA-ISH method to detect the target sequence. According to the study by Wu et al (30), there are numerous types of biomarkers for the three types CTCs. In the present study, cytokeratin 18 (CK18; epithelial biomarker), Twist (mesenchymal biomarker) and cluster of differentiation 45 (CD45; leukocyte biomarker) were utilized to distinguish epithelial, mesenchymal and hybrid CTCs. The capture probes sequences for the CK18, Twist and CD45 genes (listed in Table I) and the sequences for the bDNA signal amplification probes (listed in Table II) were purchased from Invitrogen (Thermo Fisher Scientific, Inc.). 
Table I. Capture probe sequences for the CK18, Twist, CD45 and $\mathrm{N}$-cadherin genes.

\begin{tabular}{ll}
\hline Gene & \multicolumn{1}{c}{ Sequences (5'-3') } \\
\hline CK18 & AGAAAGGACAGGACTCAGGC \\
& GAGTGGTGAAGCTCATGCTG \\
& TCAGGTCCTCGATGATCTTG \\
& CAATCTGCAGAACGATGCGG \\
& AAGTCATCAGCAGCAAGACG \\
& CTGCAGTCGTGTGATATTGG \\
Twist & ACAATGACATCTAGGTCTCC \\
& CTGGTAGAGGAAGTCGATGT \\
& CAACTGTTCAGACTTCTATC \\
& CCTCTTGAGAATGCATGCAT \\
& TTTCAGTGGCTGATTGGCAC \\
CD45 & TTACCATGGGTCCTCAATAA \\
& TCGCAATTCTTATGCGACTC \\
& TGTCATGGAGACAGTCATGT \\
& GTATTTCCAGCTTCAACTTC \\
& CCATCAATATAGCTGGCATT \\
& TTGTGCAGCAATGTATTTCC \\
& TACTTGAACCATCAGGCATC \\
& TGCATAATGCGATTTCACCA \\
N-cadherin & ACATTGAGAAGAGGCTGTCC \\
& GCTTCAGGCTCAATTTTACT \\
& TTCACTGACTCCTCAGTTAA \\
& GCTTACTGAATTGTCTTGGG \\
& TGGAGTTTTCTGGCAAGTTG
\end{tabular}

CK18, cytokeratin 18; CD45, cluster of differentiation 45 .

The abbreviated process of the RNA-ISH assay was as follows: In a 24-well plate, the cells were treated with protease at $4^{\circ} \mathrm{C}$ for $15 \mathrm{~min}$ in order to assist with to reconciling between capture probes and target sequences prior to hybridization. Following hybridization, the cells were incubated at $42^{\circ} \mathrm{C}$ for $2 \mathrm{~h}$, followed by removing the unbound probes by washing with 1,000 $\mu 1$ wash buffer (0.1X SSC; Sigma-Aldrich; Merck $\mathrm{KGaA}$ ) at $45^{\circ} \mathrm{C}$ for $5 \mathrm{~min}$ (three times). Subsequently, to achieve the signal amplification goal, the sample was incubated with $100 \mu \mathrm{l}$ preamplifier solution [30\% horse serum, $1.5 \%$ SDS, $3 \mathrm{mM}$ Tris- $\mathrm{HCl}(\mathrm{pH} 8.0$ ) and $0.5 \mathrm{fmol}$ of preamplifier (sequences are listed in Table II)] at $42^{\circ} \mathrm{C}$ for $20 \mathrm{~min}$. Following another wash with $1,000 \mu \mathrm{l}$ buffer (0.1X SSC) three times, the samples were subsequently incubated with $100 \mu \mathrm{l}$ amplifier solution [30\% horse serum, $1.5 \%$ SDS, $3 \mathrm{mM}$ Tris- $\mathrm{HCl}(\mathrm{pH} 8.0)$ and $1 \mathrm{fmol}$ of the amplifier (Table II)]. The fluorescently-labeled probes from the APEX ${ }^{\mathrm{TM}}$ Alexa Fluor ${ }^{\mathrm{TM}}$ Labeling kit (Invitrogen; Thermo Fisher Scientific, Inc.), which had been combined with the fluorescent dyes Alexa Fluor 594 (CK18), Alexa Fluor 488 (Twist), Alexa Fluor 647 (CD45) and Alexa Fluor 555 (N-cadherin), were added and incubated at $42^{\circ} \mathrm{C}$ for an additional $20 \mathrm{~min}$. Following washing with 0.1X SSC two times and staining with DAPI in turn for $5 \mathrm{~min}$ at $36^{\circ} \mathrm{C}$, the samples were analyzed with a fluorescence microscope (magnification, x100; Olympus BX53; Olympus Corporation, Tokyo, Japan). The current study defined low, medium and high $\mathrm{N}$-cadherin expression according to the following: i) Low $\mathrm{N}$-cadherin expression, $1<$ fluorescent intensity of indicated group/fluorescent intensity of control group $\leq 1.8$; ii) medium $\mathrm{N}$-cadherin expression, $1.8<$ fluorescent intensity of indicated group/fluorescent intensity of control group $\leq 2.2$; and iii) high $\mathrm{N}$-cadherin expression, $2.2<$ fluorescent intensity of indicated group/fluorescent intensity of control group.

Cell culture. The NPC cell line C666-1 was obtained from American Type Culture Collection (Manassas, VA, USA), and was cultured in Dulbecco's modified Eagle medium (DMEM; $5.5 \mathrm{mM}$ glucose) with $10 \%$ (v/v) fetal bovine serum (Gibco; Thermo Fisher Scientific, Inc.), $100 \mathrm{U} / \mathrm{ml}$ penicillin and $100 \mathrm{U} / \mathrm{ml}$ streptomycin (Invitrogen; Thermo Fisher Scientific, Inc.). The culture medium was replaced daily until the cells grew to $80 \%$ confluency.

Detection of EBV DNA. C666-1 (5x10 cells/well) cells were seeded in 6-well plates and cultured at $37^{\circ} \mathrm{C}$ in an atmosphere containing $5 \% \mathrm{CO}_{2}$ for $24 \mathrm{~h}$. C666-1 cells were then treated with $1 \mu \mathrm{mol} / 1$ XAV949 or $4 \mathrm{mg} / 1$ paclitaxel combined with $8 \mathrm{mg} / \mathrm{l}$ cisplatin for $48 \mathrm{~h}$. Subsequently, cells were analyzed with theEBV-DNA assay kit. The EBV-DNA assay was conducted according to the manufacturer's protocols of the associated ELISA kit (cat. no. YM-S0533; Yuanmu, Shanghai, China) and EBV nucleic acid quantitative detection reagent kit.

Cell viability assay. C666-1 ( $1 \times 10^{4}$ cells/well) cells were seeded in 96-well plates and cultured at $37^{\circ} \mathrm{C}$ in an atmosphere containing 5\% $\mathrm{CO}_{2}$ for $24 \mathrm{~h}$. C666-1 cells were then treated with $1 \mu \mathrm{mol} / \mathrm{l} \mathrm{XAV} 949$ or $4 \mathrm{mg} / \mathrm{l}$ paclitaxel combined with $8 \mathrm{mg} / \mathrm{l}$ cisplatin for $48 \mathrm{~h}$ at $37^{\circ} \mathrm{C}$. Subsequently, $20 \mu \mathrm{l}$ MTT solution $(5 \mathrm{mg} / \mathrm{ml})$ was added into each well and was incubated for $4 \mathrm{~h}$ at $37^{\circ} \mathrm{C}$. Following this, $150 \mu \mathrm{l} /$ well dimethyl sulfoxide was added to dissolve associated crystals. The plate was read with a scanning multi-well spectrophotometer at $570 \mathrm{~nm}$.

Western blot analysis. C666-1 cells $\left(5 \times 10^{4}\right.$ cells/well) were seeded in 6-well plates and cultured at $37^{\circ} \mathrm{C}$ in an atmosphere containing $5 \% \mathrm{CO}_{2}$ for $24 \mathrm{~h}$. Protein lysates were collected with a Membrane and Cytosol Protein Extraction kit (Beyotime Institute of Biotechnology) according to the manufacturer's protocol. Total protein was quantified using a bicinchoninic acid assay and $25 \mu \mathrm{g}$ proteins in each group were separated by $10 \%$ SDS-PAGE electrophoresis and were transferred onto polyvinylidene difluoride membranes (EMD Millipore). TBS containing 5\% skimmed milk and $0.05 \%$ Tween-20 were applied to block the membranes at $37^{\circ} \mathrm{C}$ for $2.5 \mathrm{~h}$. Finally, membranes were incubated with the primary antibodies (total caspase-3, active caspase-3, $\beta$-catenin, c-Myc and GAPDH) at $4^{\circ} \mathrm{C}$ for $14 \mathrm{~h}$ (1:500 dilution). Membranes were then incubated with the goat anti-rabbit secondary antibodies at $37^{\circ} \mathrm{C}$ for $2 \mathrm{~h}$ (1:1,000 dilution). The indicated protein bands were analyzed with a ChemiDoc XRS system (Bio-Rad Laboratories, Inc., Hercules, CA, USA) and quantified using Image J 5.0 software (National Institutes of Health, Bethesda, MD, USA). 
Table II. Sequences for the bDNA signal amplification probes.

\begin{tabular}{llll}
\hline & Function (copies) & Sequence (5'-3') & Complement (copies) \\
\hline bDNA probes for CK18 & Capture probe tail (1) & CTACAAACAAACAATATT & Preamplifier leader (1) \\
& Preamplifier repeat (5) & CGCAGCCTCAGCC & Amplifier leader (1) \\
& Amplifier repeat (5) & CCCAGACCCTACC & Label probe (1) \\
bDNA probes for Twist & Capture probe tail (1) & CTTCTCAATAACTAACAT & Preamplifier leader (1) \\
& Preamplifier repeat (5) & GACGGTCGGCGTT & Amplifier leader (1) \\
& Amplifier repeat (5) & GTCACCGCTCCAC & Label probe (1) \\
bDNA probes for CD45 & Capture probe tail (1) & CTTTATACCTTTCTTTCA & Preamplifier leader (1) \\
& Preamplifier repeat (5) & GCGCGCTGTAGGG & Amplifier leader (1) \\
& Amplifier repeat (5) & AGGCGAGGGGAGA & Label probe (1) \\
bDNA probes for N-cadherin & Capture probe tail (1) & AGCTCTTGAGGAAAAGGTCC & Preamplifier leader (1) \\
& Preamplifier repeat (5) & ACACTGTACCGCA & Amplifier leader (1) \\
& Amplifier repeat (5) & GTGCCAAGGTCGA & Label probe (1)
\end{tabular}

bDNA, branched DNA; CK18, cytokeratin 18; CD45, cluster of differentiation 45. The sequences labeled 'leader' appear once in the indicated construct, while sequences labeled 'repeat' appear the indicated number of times. The tail on the capture probe is a single sequence.

Table III. Detection of CTCs in nasopharyngeal carcinoma.

\begin{tabular}{|c|c|c|c|c|c|c|c|}
\hline \multirow[b]{2}{*}{ Clinical stage $\mathrm{a}^{\mathrm{a}}$} & \multicolumn{2}{|c|}{$\mathrm{CTC}^{+}$} & \multicolumn{2}{|c|}{ Mesenchymal CTC+ } & \multirow{2}{*}{$\begin{array}{c}\text { Median number } \\
\text { of CTCs }\end{array}$} & \multirow{2}{*}{$\begin{array}{c}\text { Mean number } \\
\text { of CTCs }\end{array}$} & \multirow[b]{2}{*}{ SD } \\
\hline & Number & PR, \% & Number & $\mathrm{PR}, \%$ & & & \\
\hline II $(n=5)$ & 5 & 100 & 3 & 60.0 & 14 & 18 & 17 \\
\hline III $(n=18)$ & 16 & 88.9 & 6 & 33.3 & 4 & 7 & 9 \\
\hline IV $(n=37)$ & 31 & 83.8 & 21 & 56.8 & 5 & 8 & 10 \\
\hline Total $(n=60)$ & 52 & 86.7 & 30 & 50.0 & 4 & 9 & 10 \\
\hline
\end{tabular}

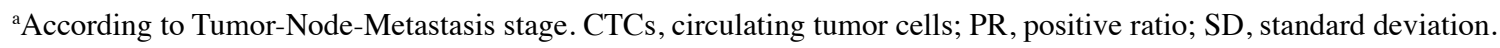

Statistical analysis. One-way analysis of variance, followed by Dunnett's multiple-comparisons test, was utilized to analyze differences among groups. The association between two variables was examined by the Spearman's rank correlation test. Kaplan-Meier analysis was used to assess the survival function. Log-rank test was used to obtain P-values of Kaplan-Meier curves. $\mathrm{P}<0.05$ was considered to indicate a statistically significant difference. Data are presented as the mean \pm standard error of the mean. All statistical analysis was performed using SPASS statistical analysis software version 19.0 (swMATH, Berlin, Germany).

\section{Results}

Detection of CTCs in NPC. A total of 60 patients who met the inclusion and exclusion criteria were enrolled in the present study. The CanPatrol ${ }^{\mathrm{TM}}$ CTC-enrichment technique was applied to isolate and analyze CTCs collected from $5 \mathrm{ml}$ peripheral blood samples of patients. During the analyses, samples were collected to analyze at different times. The number of CTCs at the first detection was classified as the baseline. At the baseline, CTCs were identified in $86.7 \%(52 / 60)$ of all patients, in which CTCs were detected in 100 (5/5), 88.9 (16/18) and
$83.8 \%(31 / 37)$ of the patients with stage II, III and IV disease, respectively. The positive ratio of mesenchymal CTCs of all patients was $50 \%(30 / 60)$, while the ratios were $60(3 / 5)$, $33.3(6 / 18)$ and $56.8 \%(21 / 37)$ in patients with stage II, III and IV disease, respectively. Furthermore, the median and mean number of CTCs present in the samples of all patients was 4 and 9, respectively (Table III). Collectively, these results indicated that CTCs could be readily detected in NPC, particularly in patients with stage II disease, demonstrating that the number of CTCs may be increased in the early stages of tumor metastasis. Nearly half of the patients expressed mesenchymal CTCs, whose positive ratio was increased.

Association of CTCs and clinical indexes. To investigate the correlation between CTCs level and clinical indexes, Spearman's $\rho$ was used for analysis. Firstly, the present study demonstrated that the number of CTCs was not associated with Tumor-Node-Metastasis (TNM) stage (33), histopathological type and grade (34), lymph node metastasis, distant metastasis, carcinoembryonic antigen (CEA) and Eastern Cooperative Oncology Group (ECOG) score (35) prior to and following therapy (data not shown). Secondly, EBV infection demonstrated a positive correlation with the total number of 
Table IV. Correlation between CTCs and EBV prior to therapy.

\begin{tabular}{lccccc}
\hline Variable & Spearman's $\rho$ & Total CTCs & Epithelial CTCs & Hybrid CTCs & Mesenchymal CTCs \\
\hline EBV & $\rho$ & 0.303 & -0.008 & 0.402 & -0.056 \\
& P-value & $0.024^{\mathrm{a}}$ & 0.956 & $0.002^{\mathrm{b}}$ & 0.683 \\
Patient samples, $\mathrm{n}$ & & 55 & 55 & 55 & 55 \\
\hline
\end{tabular}

${ }^{\mathrm{a}} \mathrm{P}<0.05,{ }^{\mathrm{b}} \mathrm{P}<0.01$ for EBV expression vs. CTCs number. CTCs, circulating tumor cells; EBV, Epstein-Barr virus.

Table V. Correlation between CTCs and ECOG score following therapy.

\begin{tabular}{|c|c|c|c|c|c|c|}
\hline Variable & Spearman's $\rho$ & Total CTCs & Epithelial CTCs & Hybrid CTCs & $\begin{array}{l}\text { Mesenchymal } \\
\text { CTCs }\end{array}$ & $\begin{array}{c}\text { PR of mesenchymal } \\
\text { CTCs }\end{array}$ \\
\hline \multirow[t]{2}{*}{ ECOG score } & $\rho$ & -0.023 & -0.013 & -0.116 & 0.258 & 0.422 \\
\hline & P-value & 0.864 & 0.922 & 0.378 & $0.046^{\mathrm{a}}$ & $0.003^{\mathrm{b}}$ \\
\hline Patient samples & & 60 & 60 & 60 & 60 & 60 \\
\hline
\end{tabular}

${ }^{\mathrm{a}} \mathrm{P}<0.05$, ECOG score vs. CTCs number; ${ }^{\mathrm{b}} \mathrm{P}<0.01$, ECOG score vs. PR of mesenchymal CTCs. CTCs, circulating tumor cells; ECOG, Eastern Cooperative Oncology Group; PR, positive ratio.

Table VI. Correlation between CTCs and therapeutic effect following treatment with cisplatin and paclitaxel.

\begin{tabular}{lccccccccc}
\hline & & & & & & Total & Epithelial \\
Variable & $\begin{array}{c}\text { Spearman's } \\
\rho\end{array}$ & $\begin{array}{c}\text { Total } \\
\text { CTCs }\end{array}$ & $\begin{array}{c}\text { Epithelial } \\
\text { CTCs }\end{array}$ & $\begin{array}{c}\text { Hybrid } \\
\text { CTCs }\end{array}$ & $\begin{array}{c}\text { Mesenchymal } \\
\text { CTCs }\end{array}$ & $\begin{array}{c}\text { CTCs } \\
\text { change }\end{array}$ & $\begin{array}{c}\text { CTCs } \\
\text { change }\end{array}$ & $\begin{array}{c}\text { CTCs } \\
\text { change }\end{array}$ & $\begin{array}{c}\text { Mesenchymal } \\
\text { CTCs change }\end{array}$ \\
\hline Therapeutic effect & $\rho$ & -0.319 & 0.019 & -0.308 & -0.494 & -0.347 & -0.025 & -0.292 & -0.279 \\
& P-value & 0.066 & 0.913 & 0.076 & $0.003^{\mathrm{b}}$ & $0.044^{\mathrm{a}}$ & 0.890 & 0.094 & 0.110 \\
Patient samples & & 34 & 34 & 34 & 34 & 34 & 34 & 34 & 34 \\
\hline
\end{tabular}

CTCs, circulating tumor cells. ${ }^{\mathrm{a}} \mathrm{P}<0.05 ;{ }^{\mathrm{b}} \mathrm{P}<0.01$.

CTCs in NPC $(\rho=0.303, \mathrm{P}<0.05$; Table IV). Furthermore, hybrid CTCs were positively associated with EBV infection $(\rho=0.402, P<0.01$; Table IV). Negative correlations between EBV infection and epithelial and mesenchymal CTCs were detected, however, these were not significant. Thirdly, ECOG score was demonstrated to be significantly associated with mesenchymal CTCs $(\rho=0.258, \mathrm{P}<0.05)$ and the proportion of mesenchymal CTCs in total CTCs following therapy $(\rho=0.422$, $\mathrm{P}<0.01$ ) (Table V). Negative correlations between ECOG score and total, epithelial, and hybrid CTCs were observed, however, these were not significant. Collectively, patients with EBV infection positive NPC were more likely to possess CTCs, particularly hybrid CTCs (Table IV). While ECOG score was positively correlated with mesenchymal CTCs following therapy (ECOG score vs. number of CTCs; $\mathrm{P}=0.046$; Table V).

Correlation with response to the therapy. To assess the effect of treatment by detecting CTCs, 60 patients were recruited, 36 of which received chemotherapy treatment with cisplatin and paclitaxel, while 18 patients were treated with cisplatin and fluoride, and 6 patients underwent radiotherapy. The treatment effects were as follows: 2/60 cases with complete response (CR), 43/60 cases with partial response (PR), 2/60 cases with stable disease (SD), 11/60 cases with progressive disease (PD) and 2/60 cases of unknown treatment effects.

In these 58 patients whose treatment effect was identifiable, $43 / 58(74.1 \%)$ cases exhibited a treatment effect associated with the changing of the number of CTCs. These included 4 cases with PD where the number of CTCs increased, 2 cases with SD where the number of CTCs did not change, 35 cases with PR where the number of CTCs were decreased or did not change, and 2 cases with CR where the number of CTCs were decreased. Similarly, 38/58 (65.5\%) cases exhibited a treatment effect associated with the change in the number of mesenchymal CTCs. For patients who were treated with cisplatin and paclitaxel, the effective rate was 76.5 and $70.6 \%$ for total and mesenchymal CTCs, respectively (data not shown). In $34 / 36$ patients who exhibited a identifiable treatment effect, the changing number of CTCs during treatment and mesenchymal CTCs following therapy demonstrated a negative correlation with the treatment effect $(\rho=-0.347, P<0.05$; and $\rho=-0.494, P<0.01$, respectively) (Table VI). However, no 
A Patient with NPC

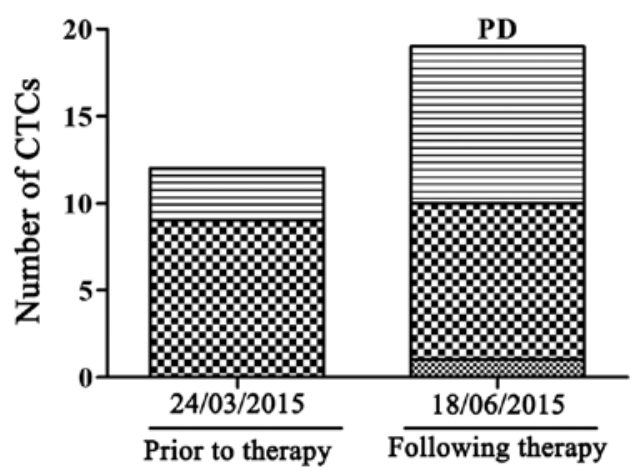

B Patient with NPC

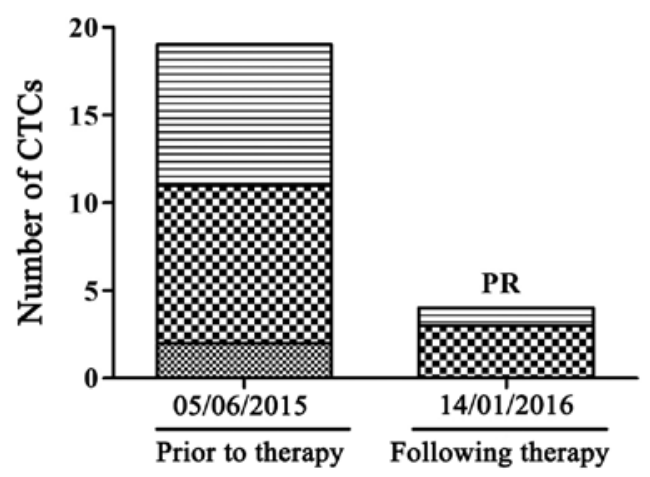

C Patient with NPC

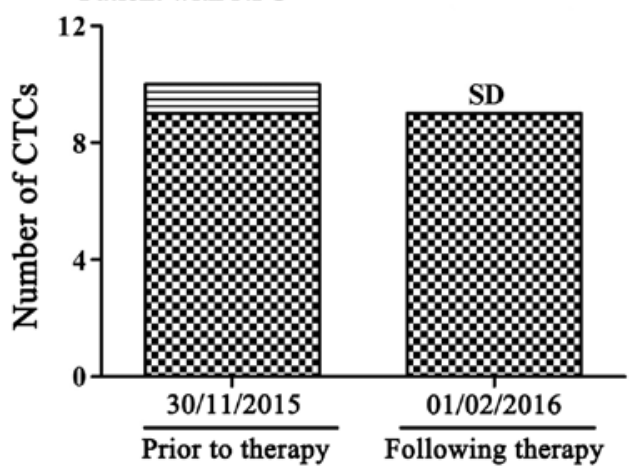

Figure 1. Change in the number of CTCs in patients with NPC prior to and following treatment with cisplatin and paclitaxel. (A) CTCs were obtained from a patient with stage IVa NPC. (B) CTCs were obtained from a separate patient with stage IVa NPC. (C) CTCs were obtained from a patient with stage IVb NPC. CTCs, circulating tumor cells; NPC, nasopharyngeal carcinoma; PD, progressive disease; PR, partial response; SD, stable disease.

significant association between therapeutic effect and number of total CTCs, epithelial CTCs or hybrid CTCs was identified in patients with NPC. Also, no significant association between therapeutic effect and change of hybrid CTCs, epithelial CTCs or mesenchymal CTCs was identified in patients with NPC. For patients who were treated with cisplatin and fluoride, the effective rate was 66.7 and $61.1 \%$ for total and mesenchymal CTCs, respectively (data not shown).

A total of three typical cases are presented to support the results. Blood samples from three patients were collected and analyzed prior to and following treatment with cisplatin and paclitaxel. A patient with stage IVa disease, whose total and mesenchymal CTCs were increased, had a PD following therapy (Fig. 1A), while another patient with stage IVa disease had a PR when the total and mesenchymal CTCs were decreased (Fig. 1B). Furthermore, a patient with stage IVb disease, whose total and mesenchymal CTCs were not significantly altered, had SD following therapy (Fig. 1C). These analyses indicated that a reduction of CTCs, particularly mesenchymal CTCs, demonstrate a curative effect.

Correlation between CTCs and progression-free survival $(P F S)$. No notable association was demonstrated between CTCs following therapy and PFS without considering the therapeutic schedule or in patients treated with cisplatin and fluoride (data not shown). However, a significant correlation was illustrated between mesenchymal CTCs following therapy and PFS in 36 patients treated with cisplatin and paclitaxel (data not shown). The PFS of patients without mesenchymal

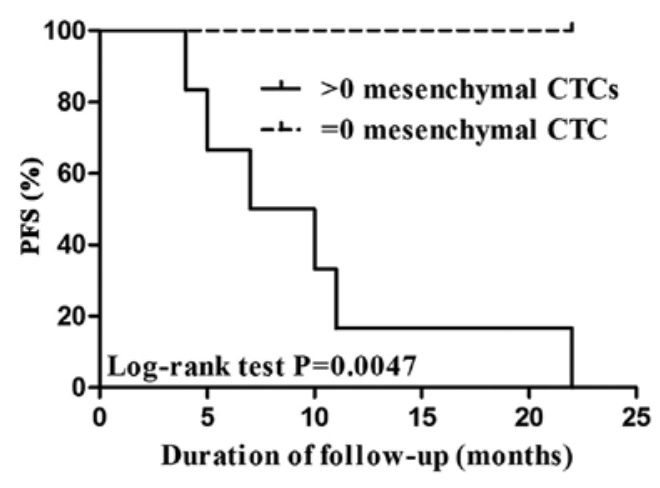

Figure 2. PFS according to CTC levels. CTCs $/ 5 \mathrm{ml}>0$ vs. $=0$. CTC, circulating tumor cell; PFS, progression free survival; CTC, circulating tumor cells.

CTCs $(\mathrm{CTC}=0)$ was significantly increased, compared with patients with $>0$ mesenchymal CTCs ( $\mathrm{P}=0.0047$; Fig. 2).

Expression of $\mathrm{N}$-cadherin in CTCs and its correlation with $N P C$. Expression of $\mathrm{N}$-cadherin is a symbol of EMT (36), however, to the best of our knowledge, $\mathrm{N}$-cadherin expression in CTCs of patients with NPC has not yet been investigated. A Tri-color RNA-ISH assay was used to indicate N-cadherin in three types of CTCs (Fig. 3). Epithelial CTCs revealed only red fluorescence due to the marker CK18. Mesenchymal CTCs demonstrated only green fluorescence due to the marker Twist. While hybrid CTCs revealed both red and green fluorescence. The $\mathrm{N}$-cadherin positive rate in epithelial, mesenchymal and 
Table VII. Association between CTCs and N-cadherin expression.

\begin{tabular}{|c|c|c|c|c|c|c|}
\hline Type of CTCs & $\begin{array}{c}\text { Cell } \\
\text { number }\end{array}$ & $\begin{array}{l}\text { N-cadherin } \\
\text { negative } \\
\text { number }(\%)\end{array}$ & $\begin{array}{l}\text { N-cadherin } \\
\text { positive } \\
\text { number }(\%)\end{array}$ & $\begin{array}{l}\text { Low } \mathrm{N} \text {-cadherin } \\
\text { expression } \\
\text { number }(\%)\end{array}$ & $\begin{array}{c}\text { Medium } \mathrm{N} \text {-cadherin } \\
\text { expression } \\
\text { number }(\%)\end{array}$ & $\begin{array}{c}\text { High } \mathrm{N} \text {-cadherin } \\
\text { expression } \\
\text { number }(\%)\end{array}$ \\
\hline Epithelial CTCs & 190 & $41(21.6)$ & $149(78.4)$ & $49(25.8)$ & $86(45.3)$ & $14(7.4)$ \\
\hline Hybrid CTCs & 1,022 & $209(20.5)$ & $813(79.5)$ & $298(29.2)$ & $434(42.5)$ & $81(7.9)$ \\
\hline Mesenchymal CTCs & 283 & $107(37.8)$ & $176(62.2)$ & $100(35.3)$ & $70(24.8)$ & $6(2.1)$ \\
\hline Total CTCs & 1,495 & 357 (23.9) & $1,138(76.1)$ & 447 (29.9) & $590(39.5)$ & $101(6.8)$ \\
\hline
\end{tabular}

CTCs, circulating tumor cells.

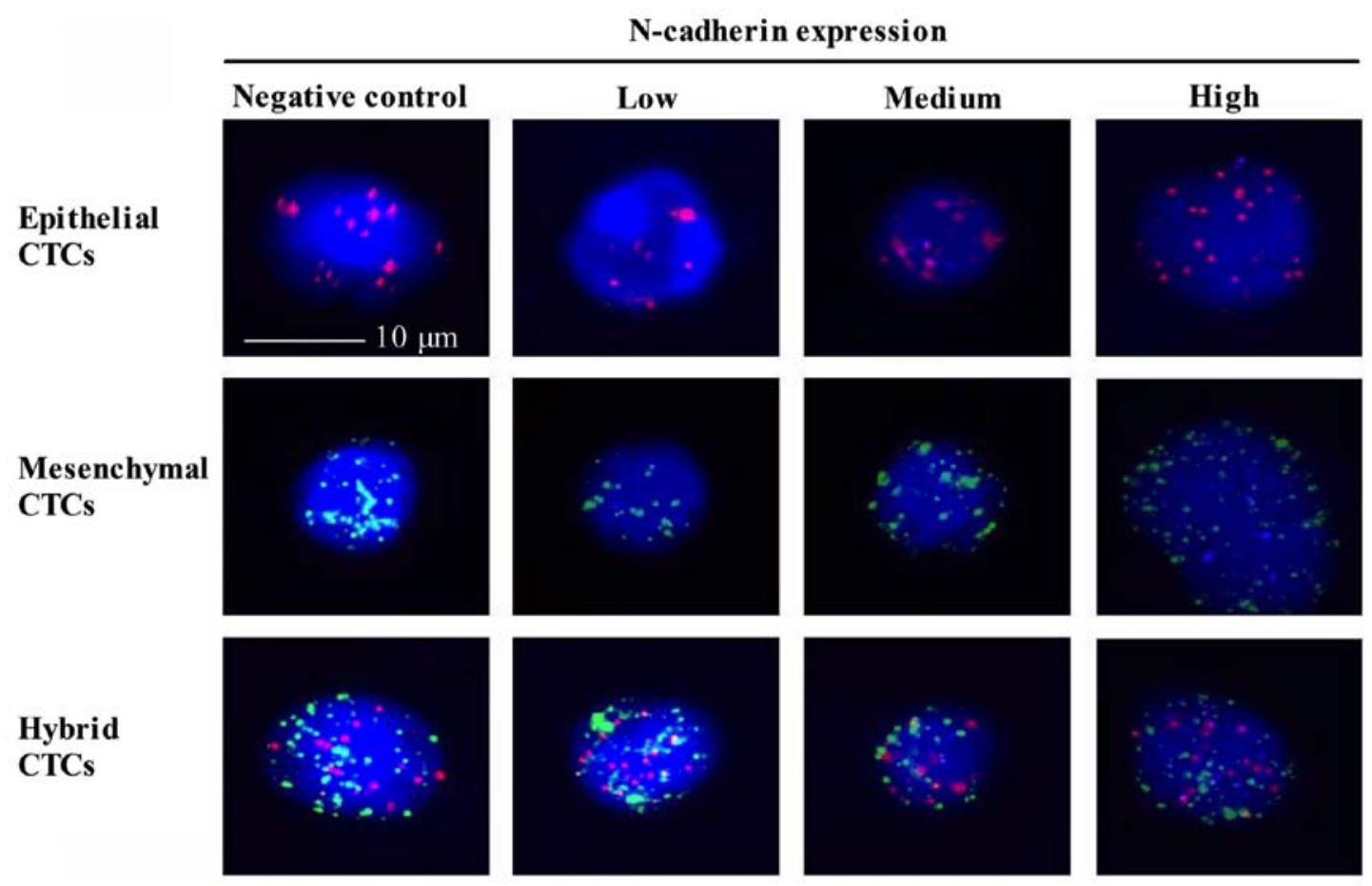

Figure 3. N-cadherin expression in different types of CTCs. N-cadherin (purple fluorescence) expression was depicted in different types of CTCs stained with CK18 (red fluorescence) and Twist (green fluorescence) biomarkers. The cells were analyzed using a x100 oil objective. CTCs, circulating tumor cells.

hybrid CTCs was 78.4, 62.2 and $79.5 \%$, respectively. Notably, the percentage of medium $\mathrm{N}$-cadherin expression in epithelial CTCs (45.3\%) and hybrid CTCs (42.5\%) was increased, compared with mesenchymal CTCs $(24.8 \%)$ (Table VII). Furthermore, high $\mathrm{N}$-cadherin expression levels in three CTCs types were consistent with the previous results. Collectively, $\mathrm{N}$-cadherin was expressed with high positive rates in all three types of CTCs. It appears that an increased N-cadherin expression was identified in CTCs with the potential of EMT, however further experiments are required to illustrate this.

Wnt/ $\beta$-catenin signaling may incite apoptosis of C666-1 cell following treatment of cisplatin combined with paclitaxel. The previous results established that the number and the marker change of CTCs indicated the effect of therapy. However, the cause of this phenomenon has not been elucidated completely. Therefore, the present study used the NPC cell line C666-1 in an attempt to clarify this mechanism.
In accordance with the change in the number of CTCs following therapy, cellular viability of C666-1 was significantly inhibited in the cisplatin combined with paclitaxel group when compared with the control group $(\mathrm{P}<0.01$; Fig. $4 \mathrm{~A})$. Furthermore, following treatment with cisplatin combined with paclitaxel, levels of EBV and N-cadherin were significantly decreased in C666-1 cells compared with the control group $(\mathrm{P}<0.01$; Fig. 4B and $\mathrm{C})$. Notably, the $\mathrm{Wnt} / \beta$-catenin inhibitor XAV949 demonstrated the identical effect on cellular viability, and EBV and N-cadherin level of C666-1 cells, indicating that Wnt/ $\beta$-catenin signaling may have a role in the regulation of C666-1 cells following treatment $(\mathrm{P}<0.01)$. By contrast, the results demonstrated that cisplatin combined with paclitaxel, exhibited a similar effect as XAV949, with significantly increased caspase-3 level and decreased Bcl2 level in C666-1 cells compared with the control group ( $\mathrm{P}<0.01$; Fig. 5A and $\mathrm{B})$, indicating that cisplatin combined with paclitaxel could result in apoptosis of C666-1 cells. Furthermore, the expression 

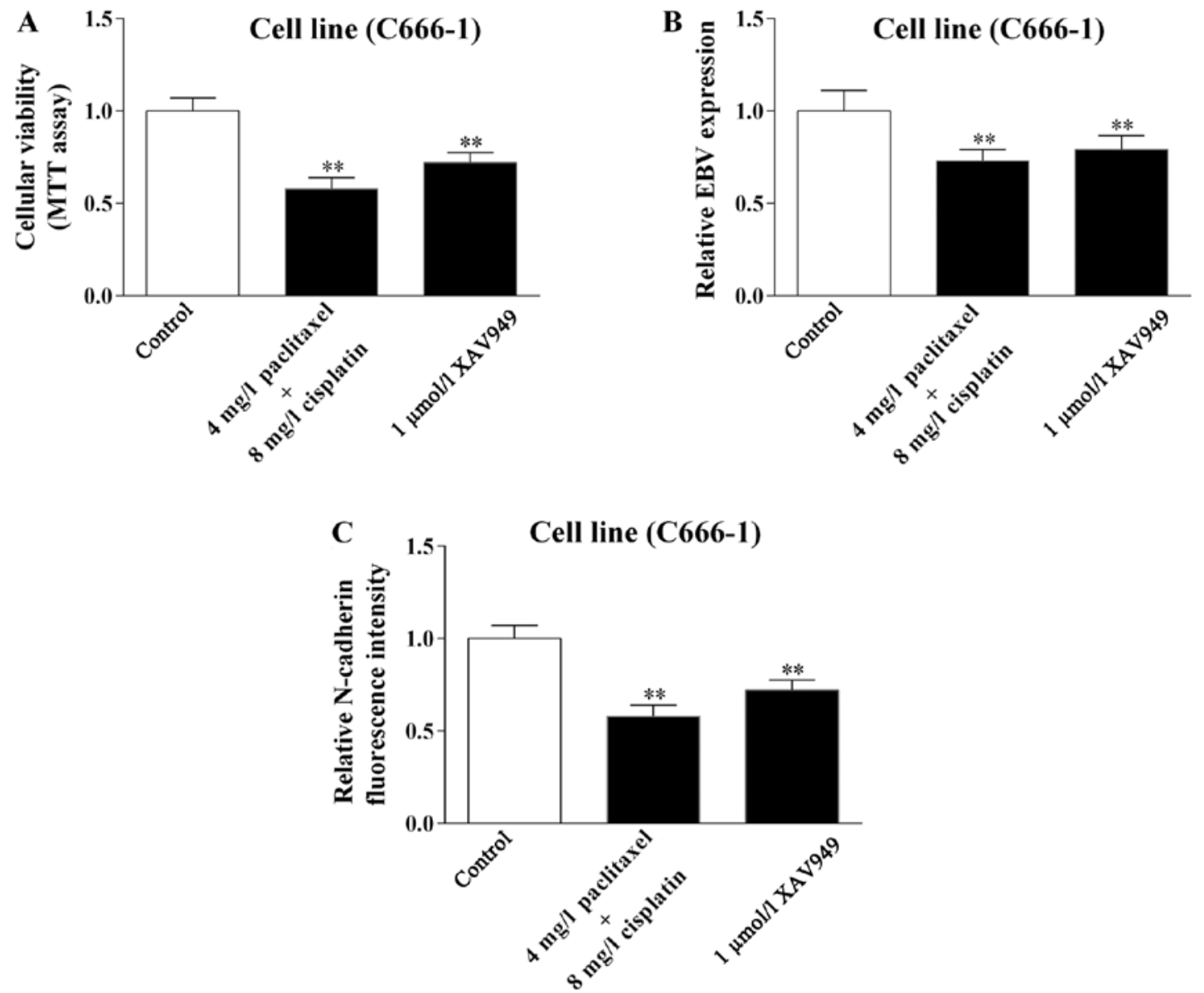

Figure 4. Effect of paclitaxel combined with cisplatin on cellular viability, and EBV and N-cadherin expression on C666-1 cells. C666-1 cells were treated with $4 \mathrm{mg} / 1$ paclitaxel combined with $8 \mathrm{mg} / \mathrm{l}$ cisplatin or $1 \mu \mathrm{mol} / 1$ XAV949 for $48 \mathrm{~h}$. (A) An MTT assay was applied to determine the change in the C666-1 cellular viability when treated with paclitaxel combined with cisplatin or XAV949. (B) An EBV-DNA assay was applied to determine the change in the C666-1 cellular EBV level when treated with paclitaxel combined with cisplatin or XAV949. (C) A Tri-color RNA-in situ hybridization assay was applied to determine the change in the C666-1 cellular N-cadherin level when treated with paclitaxel combined with cisplatin or XAV949. Data are presented as mean \pm standard error of the mean of three independent experiments. One-way analysis of variance, followed by Dunnett's multiple-comparisons test, was utilized for analyzed differences among groups. ${ }^{* *} \mathrm{P}<0.01$ vs. control group. EBV, Epstein-Barr virus.

levels of $\beta$-catenin and $c$-Myc, a key protein and downstream factor of $\mathrm{Wnt} / \beta$-catenin signaling (37), were significantly decreased by cisplatin combined with paclitaxel $(\mathrm{P}=0.008$ and $\mathrm{P}=0.006$, respectively, vs. the control) or XAV949 ( $\mathrm{P}=0.005$ and $\mathrm{P}=0.006$, respectively, vs. the control; Fig. $5 \mathrm{C}$ and $\mathrm{D}$ ). Collectively, cisplatin combined with paclitaxel could regulate the Wnt/ $\beta$-catenin signaling pathway to inhibit EBV and $\mathrm{N}$-cadherin expression, and induce apoptosis of C666-1 cells.

\section{Discussion}

Clinical staging is a basic method to evaluate tumors, and primarily depends on imaging (38). Previously, with the development of molecular targeted therapy, a growing number of molecular markers have been identified and utilized to evaluate the curative effect and prognosis of tumors (39). CTCs, from the metastasis of primary tumors, have tumor biological characteristics, and have gradually become a means to evaluate the index of a tumor (40). Monitoring the changes of CTCs in peripheral blood may be effective in evaluating the efficacy of various therapeutics. Furthermore, as techniques to detect CTCs progress, there will be a reduced requirement to perform tumor biopsies. CTCs can be used for the detection of tumors by genetic conditions, to guide targeted therapy, which is termed as 'liquid biopsy' (41). The present study demonstrated that CTCs can be identified in the peripheral blood of patients with NPC, using a technique called CanPatrol CTC enrichment, which has previously been demonstrated to effectively isolate and characterize CTCs (30).

The data obtained by the present study demonstrated that CTCs could be detected not only in advanced stages, but also in the early stages of NPC, and mesenchymal CTCs were expressed at an increased ratio. CTC positivity was not associated with clinical characters, including TNM stage, histopathological type and grade, lymph node metastasis, distant metastasis and CEA, prior to and following therapy. EBV has a high incidence of infecting CTCs, and previous research has demonstrated that EBV is expressed in all three types of CTCs (42). In the present study, the data demonstrated that EBV expression was positively associated with CTCs at a baseline level, and a high ratio of EBV can be detected in CTCs of patients with NPC at their first consultation. However, no significant correlation was identified between EBV and CTCs in patients following therapy. Notably, the present study demonstrated that the EBV level in 
A
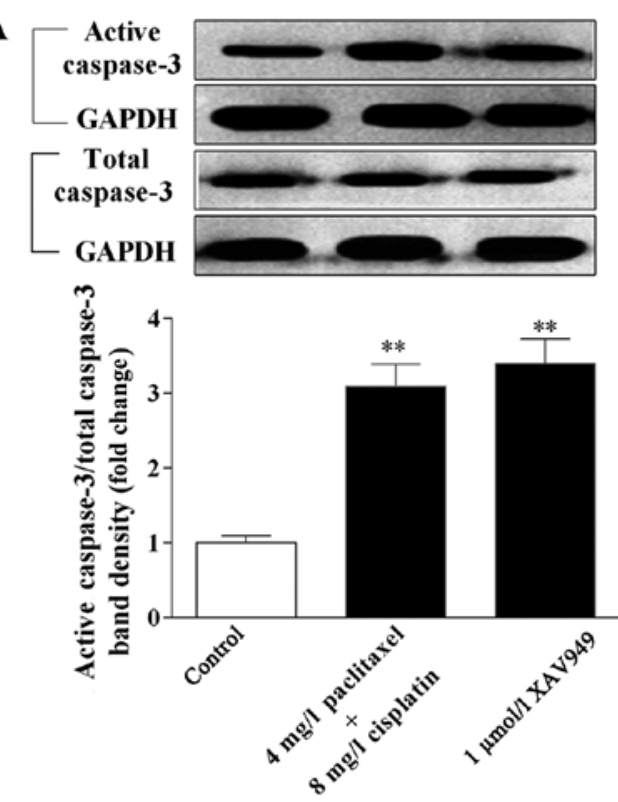

C
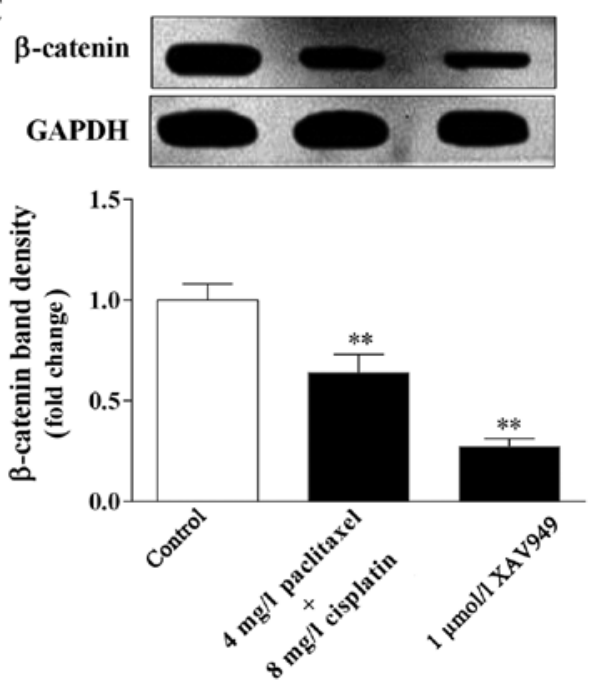

B

Bcl 2

GAPDH
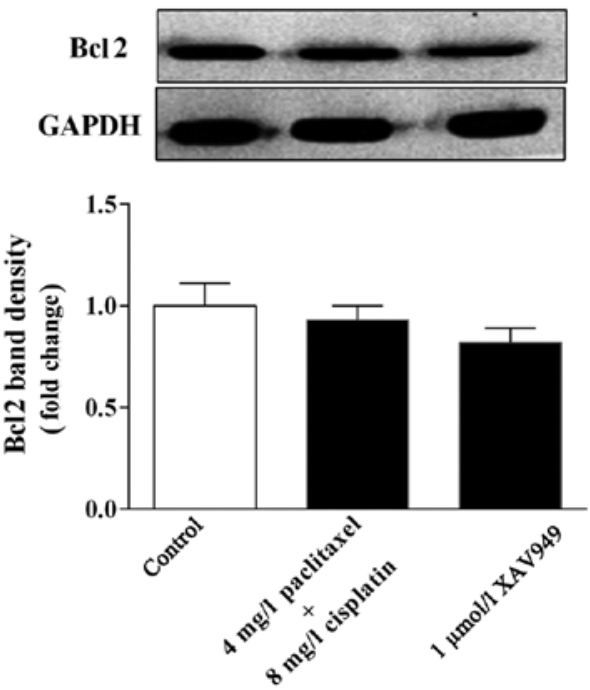

D
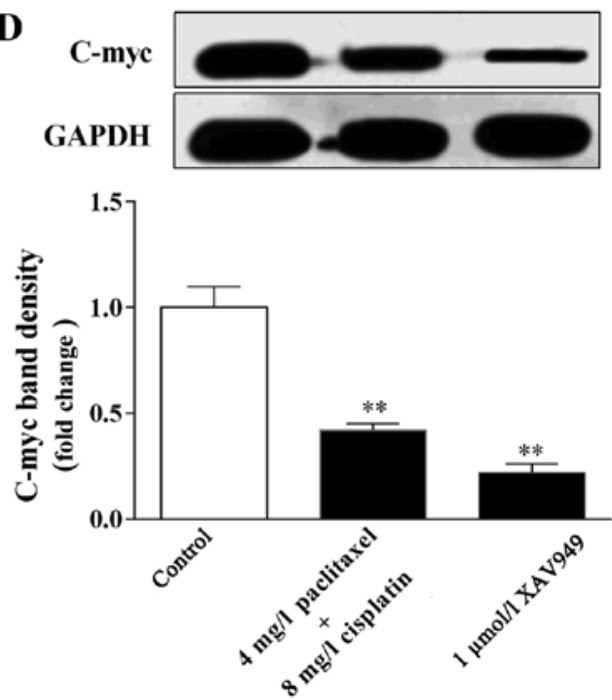

Figure 5. Effect of paclitaxel combined with cisplatin on apoptosis and Wnt/ $\beta$-catenin signaling-associated proteins expression. C666-1 cells were incubated with indicated paclitaxel combined with cisplatin or XAV949 for 48 h.Immunoblots and bar graphs of indicated proteins levels of C666-1 cells. (A) Change in total caspase-3 and active caspase-3 expression following paclitaxel following paclitaxel combined with cisplatin or XAV949 treatment. (B) Change in BCL-2 expression following paclitaxel combined with cisplatin or XAV949 treatment. (C) Change in $\beta$-catenin expression following paclitaxel combined with cisplatin or XAV949 treatment. (D) Change in c-Myc expression following paclitaxel combined with cisplatin or XAV949 treatment. Data are presented as mean \pm standard error of the mean of three independent experiments. One-way analysis of variance, followed by Dunnett's multiple-comparisons test, was utilized for analyzed differences among groups. ${ }^{* *} \mathrm{P}<0.01$ vs. control group. Bcl2, B-cell lymphoma.

C666-1 cells was decreased following treatment with paclitaxel combined with cisplatin. For the ECOG score, the results were the opposite of those for EBV, with it being negatively correlated with CTCs initially and were positively correlated following treatment. These results demonstrate that EBV may be regarded as a biomarker to diagnose NPC.

To the best of our knowledge, there are no studies regarding the association between the change in the number of CTCs and the clinical response to therapy in patients with NPC. The present data demonstrated that the increased number of total and mesenchymal CTCs following treatment indicated poor therapeutic effects. In the present study, it was determined that the number of CTCs in patients who exhibited a CR or PR following treatment was decreased or did not change. Additionally, the patients with a SD following treatment exhibited no change in the numbers of CTCs, while the number of CTCs in patients with a PD following treatment was increased. In a clinical setting, patients with NPC who initially respond to radiotherapy are frequently considered for reduction in treatment intensity or a treatment break (43). However, rapid disease progression during this treatment break is a potential disadvantage of this method (44-46). CTCs may have a role in assisting patients to prolong treatment breaks or resume therapy quicker (47). The present data demonstrated that if mesenchymal CTCs could not be detected, the PFS of patients treated with cisplatin and fluoride was prolonged, indicating that CTCs may serve as predictors of PFS.

$\mathrm{N}$-cadherin is a transmembrane, hemophilic glycoprotein belonging to the calcium-dependent cell adhesion molecule family (48). High expression of $\mathrm{N}$-cadherin is associated 
with tumor invasion and metastasis, whereas EMT is associated with tumor malignancy and metastasis, and N-cadherin is considered as a characteristic of EMT (49-51). In the present study, the expression of $\mathrm{N}$-cadherin was detected in the CTCs in 60 patients with NPC. The data demonstrated that the positive expression rate of $\mathrm{N}$-cadherin in all three types of CTCs was notably high. However, the ratio of high $\mathrm{N}$-cadherin expression in the three types of CTCs was low. Further study should prospectively address whether the expression of $\mathrm{N}$-cadherin is correlated with clinical indexes. Furthermore, the present study demonstrated that $\mathrm{N}$-cadherin was abnormally expressed in CTCs, and N-cadherin expression in C666-1 cells was significantly inhibited by treatment of paclitaxel combined with cisplatin.

In accordance with the clinical results, it was confirmed that paclitaxel combined with cisplatin could regulate $\mathrm{Wnt} / \beta$-catenin signaling to induce apoptosis and identify markers of NPC cells. These results provided a new reference to use a $\mathrm{Wnt} / \beta$-catenin signaling inhibitor combined with traditional antitumor medicine to treat NPC. However, further study should be conducted prior to any widespread clinical application.

Collectively, the results of the present study demonstrated that CTCs can be detected in the peripheral blood of patients with NPC.CTCs exhibited a statistically significant association with EBV prior to treatment, and the ECOG score following treatment. In addition, the number change of CTCs was notably associated with the treatment effect following chemotherapy, particularly in patients treated with cisplatin and paclitaxel. Paclitaxel combined with cisplatin was demonstrated to regulate $\mathrm{Wnt} / \beta$-catenin signaling to induce apoptosis and marker expression in NPC cells, and mesenchymal CTCs may serve as a predictor of PFS. These data indicated that CTCs may serve as a biomarker in monitoring the therapeutic efficacy of NPC treatments. Additional molecular mechanism-based studies are required in order to confirm whether a signaling inhibitor combined with traditional antitumor medicine could be an effective treatment for NPC.

\section{Acknowledgements}

The authors would like to thank Guangzhou SurExam Bio-Tech Co., Ltd. (Guangzhou, China), who supported the present study with CTC isolation and Tri color RNA ISH assay experiments.

\section{Funding}

Not applicable.

\section{Authors' contributions}

Study design was undertaken by ZL and PY. The clinical studies were conducted by DX. The experimental studies were performed by ZL, HC and ZW. Data acquisition was performed by YY, SW and ZW. Data analysis was performed by $\mathrm{CL}, \mathrm{ZC}, \mathrm{SW}, \mathrm{DL}$ and $\mathrm{ZW}$. Interpretation of data was performed by ZW. Literature research was performed by PY, SW and DL. Revision of the manuscript was performed by ZL, DL, ZW and PY. Manuscript preparation was conducted by ZL. Manuscript editing was conducted by ZW and ZL.

\section{Availability of data and materials}

The datasets used or analyzed in the study are available from the corresponding author on reasonable request. We declared that materials described in the manuscript, including all relevant raw data, will be freely available to any scientist wishing to use them for non-commercial purposes, without breaching participant confidentiality.

\section{Ethics approval and consent to participate}

The present study was approved by the Ethical Committee of the People's Hospital of Gaozhou (Gaozhou, China). Patients provided written informed consent for inclusion in the present study.

\section{Patient consent for publication}

Not applicable.

\section{Competing interests}

The authors declare that they have no competing interests.

\section{References}

1. Cao SM, Simons MJ and Qian CN: The prevalence and prevention of nasopharyngeal carcinoma in China. Chin J Cancer 30: 114-119, 2011.

2. Horikawa T, Yoshizaki T, Kondo S, Furukawa M, Kaizaki Y and Pagano JS: Epstein-Barr Virus latent membrane protein 1 induces Snail and epithelial-mesenchymal transition in metastatic nasopharyngeal carcinoma. Br J Cancer 104: 1160-1167, 2011.

3. Chan AT: Nasopharyngeal carcinoma. Ann Oncol 21 (Suppl 7): vii308-vii312, 2010.

4. Lin JH, Jiang CQ, Ho SY, Zhang WS, Mai ZM, Xu L, Lo CM and Lam TH: Smoking and nasopharyngeal carcinoma mortality: A cohort study of 101,823 adults in Guangzhou, China. BMC Cancer 15: 906, 2015.

5. Sousa H, Mesquita L, Ribeiro J, Catarino R, Breda E and Medeiros R: Polymorphisms in host immune response associated genes and risk of nasopharyngeal carcinoma development in Portugal. Immunobiology 221: 145-152, 2016.

6. Ashworth TR: A case of cancer in which cells similar to those in the tumours were seen in the blood after death. Aust Med J 14: 146-149, 1869.

7. Gupta GP and Massagué J: Cancer metastasis: building a framework. Cell 127: 679-695, 2006.

8. Książkiewicz M, Markiewicz A and Żaczek AJ: Epithelial-mesenchymal transition: A hallmark in metastasis formation linking circulating tumor cells and cancer stem cells. Pathobiology 79 : 195-208, 2012.

9. Guarino M: Epithelial-mesenchymal transition and tumour invasion. Int J Biochem Cell Biol 39: 2153-2160, 2007.

10. Lecharpentier A, Vielh P, Perez-Moreno P, Planchard D, Soria JC and Farace F: Detection of circulating tumour cells with a hybrid (epithelial/mesenchymal) phenotype in patients with metastatic non-small cell lung cancer. Br J Cancer 105: 1338-1341, 2011.

11. Economopoulou P, Georgoulias V and Kotsakis A: Classifying circulating tumor cells to monitor cancer progression. Expert Rev Mol Diagn 17: 153-165, 2017.

12. Cai LL, Ye HM, Zheng LM, Ruan RS and Tzeng CM: Circulating tumor cells (CTCs) as a liquid biopsy material and drug target. Curr Drug Targets 15: 965-972, 2014.

13. Cristofanilli M: Circulating tumor cells, disease progression, and survival in metastatic breast cancer. Semin Oncol 33 (3 Suppl 9): S9-S14, 2006.

14. Nolé F, Munzone E, Zorzino L, Minchella I, Salvatici M, Botteri E, Medici M, Verri E, Adamoli L, Rotmensz N, et al: Variation of circulating tumor cell levels during treatment of metastatic breast cancer: Prognostic and therapeutic implications. Ann Oncol 19: 891-897, 2008. 
15. Cohen SJ, Punt CJ, Iannotti N, Saidman BH, Sabbath KD, Gabrail NY, Picus J, Morse M, Mitchell E, Miller MC, et al: Relationship of circulating tumor cells to tumor response, progression-free survival, and overall survival in patients with metastatic colorectal cancer. J Clin Oncol 26: 3213-3221, 2008.

16. Fina E, Necchi A, Giannatempo P, Colecchia M, Raggi D, Daidone MG and Cappelletti V: Clinical significance of early changes in circulating tumor cells from patients receiving first-line cisplatin-based chemotherapy for metastatic urothelial carcinoma. Bladder Cancer 2: 395-403, 2016.

17. Hirose T, Oki Y, Kusumoto S, Sugiyama T, Shirai T, Nakashima M, Yamaoka T, Okuda K, Ohnishi T, Ohmori T and Adachi M: Circulating tumor cells as a predictive marker for chemotherapy and prognostic marker in patients with metastatic non-small cell lung cancer. J Clin Oncol 29 (Suppl 15): e21020, 2011.

18. Hall C, Valad L and Lucci A: Circulating tumor cells in breast cancer patients. Crit Rev Oncog 21: 125-139, 2016.

19. Alva A, Friedlander T, Clark M, Huebner T, Daignault S, Hussain M, Lee C, Hafez K, Hollenbeck B, Weizer A, et al: Circulating tumor cells as potential biomarkers in bladder cancer. J Urol 194: 790-798, 2015.

20. Derycke LD and Bracke ME: N-cadherin in the spotlight of cell-cell adhesion, differentiation, embryogenesis, invasion and signalling. Int J Dev Bio 48: 463-476, 2004.

21. Craig SE and Brady-Kalnay SM: Cancer cells cut homophilic cell adhesion molecules and run. Cancer Res 71: 303-309, 2011.

22. Nieman MT, Prudoff RS, Johnson KR and Wheelock MJ: $\mathrm{N}$-cadherin promotes motility in human breast cancer cells regardless of their E-cadherin expression. J Cell Biol 147: 631-644, 1999.

23. Araki K, Shimura T, Suzuki H, Tsutsumi S, Wada W, Yajima T, Kobayahi T, Kubo N and Kuwano H: E/N-cadherin switch mediates cancer progression via TGF- $\beta$-induced epithelial-to-mesenchymal transition in extrahepatic cholangiocarcinoma. Br J Cancer 105: 1885-1893, 2011.

24. Li S and Jiao J: Effects of N-cadherin expression on cell cycle, cell apoptosis and invasiveness and metastasis of tongue squamous cell carcinoma cell line Tca8113 cells. Zhonghua Kou Qiang Yi Xue Za Zhi 46: 365-369, 2011 (In Chinese).

25. Hazan RB, Phillips GR, Qiao RF, Norton L and Aaronson SA Exogenous expression of $\mathrm{N}$-cadherin in breast cancer cells induces cell migration, invasion, and metastasis. J Cell Biol 148 779-790, 2000

26. Valkenburg KC, Graveel CR, Zylstra-Diegel CR, Zhong Z and Williams BO: Wnt/ $\beta$-catenin signaling in normal and cancer stem cells. Cancers (Basel) 3: 2050-2079, 2011.

27. Chen K, Huang $\mathrm{Y}$ and Chen J: Understanding and targeting cancer stem cells: Therapeutic implications and challenges. Acta Pharmacol Sin 34: 732-740, 2013.

28. Lin QQ, Jin-Tian LI and Wang M: Role of Wnt/ $\beta$-catenin pathway in differentiation of nasopharyngeal carcinoma. J Sun Yat-sen Univ (Med Sci) 4: 384-387, 2005 (In Chinese).

29. Wu S, Liu Z, Liu S, Lin L, Yang W and Xu J: Enrichment and enumeration of circulating tumor cells by efficient depletion of leukocyte fractions. Clin Chem Lab Med 52: 243-251, 2014.

30. Wu S, Liu S, Liu Z, Huang J, Pu X, Li J, Yang D, Deng H, Yang $\mathrm{N}$ and $\mathrm{Xu} \mathrm{J}$ : Classification of circulating tumor cells by epithelial-mesenchymal transition markers. PLoS One 10 e0123976, 2015.

31. Tsongalis GJ: Branched DNA technology in molecular diagnostics. Am J Clin Pathol 126: 448-453, 2006.

32. Horn T, Chang CA and Urdea MS: Chemical synthesis and characterization of branched oligodeoxyribonucleotides (bDNA) for use as signal amplifiers in nucleic acid quantification assays. Nucleic Acids Res 25: 4842-4849, 1997.

33. Chan AT: Nasopharyngeal carcinoma. Ann Oncol 21 (Suppl 7): vii308-vii312, 2010.

34. Wei KR, Xu Y, Liu J, Zhang WJ and Liang ZH: Histopathological classification of nasopharyngeal carcinoma. Asian Pac J Cancer Prev 12: 1141-1147, 2011.
35. Hui EP, Ma BB, King AD, Mo F, Chan SL, Kam MK, Loong HH, Ahuja AT, Zee BC and Chan AT: Hemorrhagic complications in a phase ii study of sunitinib in patients of nasopharyngeal carcinoma who has previously received high-dose radiation. Ann Oncol 22: 1280-1287, 2011.

36. Luo WR, Wu AB, Fang WY, Li SY and Yao KT: Nuclear expression of N-cadherin correlates with poor prognosis of nasopharyngeal carcinoma. Histopathology 61: 237-246, 2012.

37. Shi L, Wu YX, Yu JH, Chen X, Luo XJ and Yin YR: Research of the relationship between $\beta$-catenin and c-myc-mediated Wnt pathway and laterally spreading tumors occurrence. Eur Rev Med Pharmacol Sci 21: 252-257, 2017.

38. OuYang PY, Su Z, Ma XH, Mao YP, Liu MZ and Xie FY: Comparison of TNM staging systems for nasopharyngeal carcinoma, and proposal of a new staging system. Br J Cancer 109: 2987-2997, 2013

39. Mehta S, Shelling A, Muthukaruppan A, Lasham A, Blenkiron C, Laking $\mathrm{G}$ and Print $\mathrm{C}$ : Predictive and prognostic molecular markers for cancer medicine. Ther Adv Med Oncol 2: 125-148, 2010.

40. Micalizzi DS, Haber DA and Maheswaran S: Cancer metastasis through the prism of epithelial-to-mesenchymal transition in circulating tumor cells. Mol Oncol 11: 770-780, 2017.

41. Alix-Panabieres $C$ and Pantel K: The circulating tumor cells: Liquid biopsy of cancer. Klin Lab Diagn: 60-64, 2014 (In Russian).

42. Si Y, Lan G, Deng Z, Wang Y, Lu Y, Qin Y, Huang B, Yang Y, Weng J, Han X, et al: Distribution and clinical significance of circulating tumor cells in nasopharyngeal carcinoma. Jpn J Clin Oncol 46: 622-630, 2016.

43. Chen X and Tang Q: The impact of radiotherapy course length on the treatment results of nasopharyngeal carcinoma (NPC). Chin J Cancer Res 7: 130-133, 1995.

44. Ren JH, Dai XF, Yan GL, Jin M, Liu CW, Yang KY, Wu G and Ma CM: Acute oral mucositis in nasopharyngeal carcinoma patients treated with radiotherapy: association with genetic polymorphism in DNA DSB repair genes. Int J Radiat Biol 90: 256-261, 2014.

45. Lengyel E, Baricza K, Somogyi A, Olajos J, Pápai Z, Godény $M$, Németh $G$ and Esik O: Reirradiation of locally recurrent nasopharyngeal carcinoma. Strahlenther Onkol 179: 298-305, 2003.

46. Kong FF, Ying H, Du CR, Huang S, Zhou JJ and Hu CS: Effectiveness and toxicities of intensity-modulated radiation therapy for patients with T4 nasopharyngeal carcinoma. PLoS One 9: e91362, 2014.

47. Paterlini-Brechot $\mathrm{P}$ and Benali NL: Circulating tumor cells (CTC) detection: Clinical impact and future directions. Cancer Lett 253: 180-204, 2007.

48. Hatta K, Nose A, Nagafuchi A and Takeichi M: Cloning and expression of cDNA encoding a neural calcium-dependent cell adhesion molecule: Its identity in the cadherin gene family. J Cell Biol 106: 873-881, 1988

49. MicalizziDS,FarabaughSMandFordHL:Epithelial-mesenchymal transition in cancer: Parallels between normal development and tumor progression. J Mammary Gland Biol Neoplasia 15: 117-134, 2010.

50. Iwatsuki M, Mimori K, Yokobori T, Ishi H, Beppu T, Nakamori S, Baba $\mathrm{H}$ and Mori M: Epithelial-mesenchymal transition in cancer development and its clinical significance. Cancer Sci 101: 293-299, 2010

51. Drocaş AI, Tomescu PI, Mitroi G, Drăgoescu PO, Mărgăritescu C, Stepan AE, Surlin V, CrăiŢoiu S, Drocas I, Ungureanu AM, et al: The cadherin switch assessment in the epithelial-mesenchymal transition of urothelial bladder carcinomas. Rom J Morphol Embryol 57: 1037-1044, 2016. 